\title{
Guaranteed Cost Control of Uncertain Differential Linear Repetitive Processes
}

\author{
Wojciech Paszke, Krzysztof Galkowski, Eric Rogers, and David H. Owens
}

\begin{abstract}
This paper deals with the problem of designing a control law for differential linear repetitive processes based on minimizing a cost function in the presence of uncertainties in the process model. This control law results in a closed-loop stable process with an associated cost function which is bounded for all admissible uncertainties. Moreover, an optimization algorithm is developed to design this law such that it minimizes the upperbound of the closed-loop cost function.
\end{abstract}

Index Terms-Differential repetitive processes, guaranteed cost control, two-dimensional (2-D) systems.

\section{INTRODUCTION}

$\mathbf{L}$ INEAR repetitive processes are a distinct class of two-dimensional (2-D) systems (i.e., information propagation in two independent directions) of both systems' theoretic and applications interest. Physical examples of repetitive processes include long-wall coal cutting and metal rolling operations (see, for example, [7]). Also, in recent years, applications have arisen where adopting a repetitive process setting for analysis has distinct advantages over alternatives. Examples of these so-called algorithmic applications include classes of iterative learning control (ILC) schemes [1] and iterative algorithms for solving nonlinear dynamic optimal control problems based on the maximum principle [6]. In the case of ILC for the linear dynamics case, the stability theory for so-called differential and discrete linear repetitive processes is the essential basis for the development of a rigorous stability/convergence theory for one class of very powerful algorithms. For the nonlinear optimal control application, the repetitive process setting for analysis has provided numerically robust and computationally feasible solution algorithms.

The processes cannot be controlled by direct extension of existing techniques from standard [termed one-dimensional (1-D) here] systems theory/algorithms because such an approach ignores their inherent 2-D systems structure, i.e., information propagation occurs from pass-to-pass and along a given pass and the initial conditions are reset before the start of the next

Manuscript received March 23, 2004. This paper was recommended by Associate Editor X. Xia.

W. Paszke and K. Galkowski are with the Institute of Control and Computation Engineering, University of Zielona Góra, 65-246 Zielona Góra, Poland (e-mail: w.paszke@issi.uz.zgora.pl; k.galkowski@issi.uz.zgora.pl).

E. Rogers is with the School of Electronics and Computer Science, University of Southampton, Southampton SO17 1BJ, U.K. (e-mail: etar@ecs.soton.ac.uk).

D. H. Owens is with Department of Automatic Control and Systems Engineering, University of Sheffield, Sheffield S1 3JD, U.K. (e-mail: d.h.owens@sheffield.ac.uk).

Digital Object Identifier 10.1109/TCSII.2004.836057 pass. Also, the finite pass length (and hence information propagation in this direction only occurs over a finite duration) is the key difference with other classes of 2-D linear systems. Moreover, for the differential linear repetitive processes considered here, the dynamics in the along-the-pass direction are governed by a matrix linear differential equation and hence they cannot be controlled by any of the techniques/algorithms developed for 2-D discrete linear systems, such as those described by the extensively studied Roesser and Fornasini-Marchesini models (the original references for these models can be found for, example, in [7]).

Clearly, there is a need to develop a systems theory for these processes for onward translation (where appropriate) into numerically reliable design algorithms. This general area has been the subject of substantial work but the currently available robust stability and stabilization results [2] do not include any performance criteria in the design of the control law.

In this paper, we develop a solution to the so-called guaranteed cost control problem for differential linear repetitive processes (for the discrete 2-D linear systems case, see [3]). The solution gives a control law which ensures an adequate level of performance as represented by the cost function. Based on the state-space model description of the dynamics, the conditions which guarantee stability and the existence of the guaranteed cost control law are developed in terms of the feasibility of linear matrix inequalities (LMIs). These inequalities, in turn, can be solved using well-established effective numerical algorithms [5]. Finally, an optimization algorithm is developed which minimizes the upperbound on the cost function.

Throughout this paper, the null matrix and the identity matrix with the required dimensions are denoted by 0 and $I$, respectively. Moreover, $M>0$ (respectivley, $M<0$ ) denotes a matrix $M$ which is real symmetric and positive (respectively, negative) definite. We also use $(\star)$ to denote the transpose of matrix blocks in some of the LMIs employed (which are required to be symmetric). The following lemma is required in the proofs of some of the results developed here.

Lemma 1: [4] Let $\Sigma_{1}$ and $\Sigma_{2}$ be real matrices of appropriate dimensions. Then, for any matrix $\mathcal{F}$ satisfying $\mathcal{F}^{T} \mathcal{F} \leq I$ and a scalar $\epsilon>0$ the following inequality holds:

$$
\Sigma_{1} \mathcal{F} \Sigma_{2}+\Sigma_{2}^{T} \mathcal{F} \Sigma_{1}^{T} \leq \epsilon^{-1} \Sigma_{1} \Sigma_{1}^{T}+\epsilon \Sigma_{2}^{T} \Sigma_{2}
$$

Also, we will make extensive use of the well-known Schur complement formula for matrices. 


\section{Preliminaries And Problem Statement}

Consider uncertain differential linear repetitive processes with pass length $\alpha<\infty$ described by the following state space model over $0 \leq t \leq \alpha, k \geq 0$ :

$$
\begin{aligned}
\dot{x}_{k+1}(t)= & (A+\Delta A) x_{k+1}(t)+\left(B_{0}+\Delta B_{0}\right) y_{k}(t) \\
& +(B+\Delta B) u_{k+1}(t) \\
y_{k+1}(t)= & (C+\Delta C) x_{k+1}(t)+\left(D_{0}+\Delta D_{0}\right) y_{k}(t) \\
& +(D+\Delta D) u_{k+1}(t) .
\end{aligned}
$$

where, on pass $k, x_{k}(t) \in \mathcal{R}^{n}$ is the state vector, $y_{k}(t) \in \mathcal{R}^{m}$ is the pass profile vector, and $u_{k}(t) \in \mathcal{R}^{l}$ is the vector of control inputs. The matrices $A, B, B_{0}, C, D$, and $D_{0}$ define the nominal model and $\Delta A, \Delta B, \Delta B_{0}, \Delta C, \Delta D$, and $\Delta D_{0}$ represent admissible uncertainties which are assumed to be of the form

$$
\left[\begin{array}{ccc}
\Delta A & \Delta B_{0} & \Delta B \\
\Delta C & \Delta D_{0} & \Delta D
\end{array}\right]=\left[\begin{array}{l}
H_{1} \\
H_{2}
\end{array}\right] \mathcal{F}\left[\begin{array}{lll}
E_{1} & E_{2} & E_{3}
\end{array}\right]
$$

In this last equation, $H_{1}, H_{2}, E_{1}, E_{2}$, and $E_{3}$ are known constant matrices of compatible dimensions, and $\mathcal{F}$ is an unknown matrix with constant entries which satisfies

$$
\mathcal{F}^{T} \mathcal{F} \leq I \text {. }
$$

To complete the process description, it is necessary to specify the boundary conditions, i.e., the state initial vector on each pass and the initial pass profile (i.e., on pass 0 ). The simplest possible choice for these is

$$
\begin{aligned}
x_{k+1}(0) & =d_{k+1}, \quad k \geq 0 \\
y_{0}(t) & =f(t)
\end{aligned}
$$

where the vector $d_{k+1} \in \mathcal{R}^{n}$ has known constant entries and $f(t) \in \mathcal{R}^{m}$ is the vector whose entries are known functions of $t$ over $0 \leq t \leq \alpha$. For ease of presentation, we will make no further explicit reference to the boundary conditions in this paper.

The stability theory [7] for linear repetitive processes consists of two distinct concepts, but here it is the stronger of these which is required. This is termed stability along the pass and several equivalent sets of necessary and sufficient conditions for processes described by (2) to have this property are known. Moreover, it is possible to give a physical interpretation of this property (see again [7]). In this study, stability along the pass for all admissible uncertainties, also termed robustly stable, will be characterized by the following result.

Theorem 1: [2] A differential linear repetitive process described by (2) is robustly stable if there exist matrices $P_{1}>0$, $P_{2}>0$, such that (6), shown at the bottom of the page, holds.

Many applications will require a control law which not only guarantees stability along the pass but also meets specified per- formance criteria. This is an area for which no results currently exist, and here we develop a solution to the problem of obtaining a control law which simultaneously robustly stabilizes a process described by (2) and guarantees that the associated cost function, defined by

$$
\begin{aligned}
J & =\sum_{k=0}^{\infty} \int_{0}^{\infty}\left(u_{k+1}^{T}(t) \Psi u_{k+1}(t)\right) d t \\
& +\sum_{k=0}^{\infty} \int_{0}^{\infty}\left(\left[\begin{array}{c}
x_{k+1}(t) \\
y_{k}(t)
\end{array}\right]^{T}\left[\begin{array}{cc}
Q_{1} & 0 \\
0 & Q_{2}
\end{array}\right]\left[\begin{array}{c}
x_{k+1}(t) \\
y_{k}(t)
\end{array}\right]\right) d t
\end{aligned}
$$

where $\Psi>0, Q_{1}>0$, and $Q_{2}>0$ are given matrices, is bounded for all admissible uncertainties (3).

Remark 1: Repetitive processes are defined over the finite pass length $\alpha$ and, in practice, only a finite number of passes, say $p$, will actually be completed. Hence, the cost function (7) should be replaced by

$$
\begin{aligned}
J & =\sum_{k=0}^{p} \int_{0}^{\alpha}\left(u_{k+1}^{T}(t) \Psi u_{k+1}(t)\right) d t \\
& +\sum_{k=0}^{p} \int_{0}^{\alpha}\left(\left[\begin{array}{c}
x_{k+1}(t) \\
y_{k}(t)
\end{array}\right]^{T}\left[\begin{array}{cc}
Q_{1} & 0 \\
0 & Q_{2}
\end{array}\right]\left[\begin{array}{c}
x_{k+1}(t) \\
y_{k}(t)
\end{array}\right]\right) d t .
\end{aligned}
$$

However, it is routine to argue that the signals involved can be extended from $[0, \alpha]$ to the infinite interval in such a way that projection of the infinite interval solution onto the finite interval is possible. The same is true for the pass-to-pass direction, and hence we will work with (7).

We start by developing the LMI condition which guarantees that the unforced (the control input terms are deleted) process is stable along the pass and the associated cost function is bounded for all admissible uncertainties. These results are then extended to design a guaranteed cost controller.

\section{Guaranteed Cost Bound}

In this section, we are interested in finding an upperbound for corresponding cost function of the unforced process $\left(u_{k+1}(t)=\right.$ $0)$

$$
\left[\begin{array}{c}
\dot{x}_{k+1}(t) \\
y_{l+1}(t)
\end{array}\right]=\left(\left[\begin{array}{cc}
A & B_{0} \\
C & D_{0}
\end{array}\right]+\left[\begin{array}{cc}
\Delta A & \Delta B_{0} \\
\Delta C & \Delta D_{0}
\end{array}\right]\right)\left[\begin{array}{c}
x_{k+1}(t) \\
y_{k}(t)
\end{array}\right]
$$

with the associated cost function

$$
J_{0}=\sum_{k=0}^{\infty} \int_{0}^{\infty}\left(\left[\begin{array}{c}
x_{k+1}(t) \\
y_{k}(t)
\end{array}\right]^{T}\left[\begin{array}{cc}
Q_{1} & 0 \\
0 & Q_{2}
\end{array}\right]\left[\begin{array}{c}
x_{k+1}(t) \\
y_{k}(t)
\end{array}\right]\right) d t .
$$

The following theorem gives a sufficient condition for stability along the pass with guaranteed cost.

$$
\left[\begin{array}{ccc}
-P_{2} & (\star) & (\star) \\
(C+\Delta C)^{T} P_{2} & (A+\Delta A)^{T} P_{1}+P_{1}(A+\Delta A) & (\star) \\
\left(D_{0}+\Delta D_{0}\right)^{T} P_{2} & \left(B_{0}+\Delta B_{0}\right)^{T} P_{1} & -P_{2}
\end{array}\right]<0
$$


Theorem 2: An unforced differential linear repetitive process described by (2) is robustly stable if there exist matrices $P_{1}>0$, $P_{2}>0$, and a scalar $\epsilon>0$ such that LMI given in (11), shown at the bottom of the page, holds. Moreover, in this case, the cost function (10) satisfies the following upperbound:

$$
J_{0} \leq \sum_{k=0}^{\infty} x_{k+1}^{T}(0) P_{1} x_{k+1}(0)+\int_{0}^{\infty} y_{0}^{T}(t) P_{2} y_{0}(t) d t
$$

Proof: Define the matrices

$$
\begin{array}{ll}
A_{1}=\left[\begin{array}{cc}
A & B_{0} \\
0 & 0
\end{array}\right], & \Delta A_{1}=\left[\begin{array}{cc}
\Delta A & \Delta B_{0} \\
0 & 0
\end{array}\right] \\
A_{2}=\left[\begin{array}{cc}
0 & 0 \\
C & D_{0}
\end{array}\right], & \Delta A_{2}=\left[\begin{array}{cc}
0 & 0 \\
\Delta C & \Delta D_{0}
\end{array}\right]
\end{array}
$$

and the vectors

$$
\xi(k, t)=\left[\begin{array}{c}
\dot{x}_{k+1}(t) \\
y_{k+1}(t)
\end{array}\right], \quad \zeta(k, t)=\left[\begin{array}{c}
x_{k+1}(t) \\
y_{k}(t)
\end{array}\right] .
$$

Then rewrite (9) as

$$
\xi(k, t)=\left(\left(A_{1}+\Delta A_{1}\right)+\left(A_{2}+\Delta A_{2}\right)\right) \zeta(k, t)
$$

and choose the candidate Lyapunov function as

$$
\begin{aligned}
V(k, t) & :=V_{1}(k, t)+V_{2}(k, t) \\
& =x_{k+1}^{T}(t) P_{1} x_{k+1}(t)+y_{k}^{T}(t) P_{2} y_{k}(t)
\end{aligned}
$$

where $P_{1}>0$ and $P_{2}>0$. (This function is a combination of two independent indeterminates due to the 2-D nature of the repetitive processes considered here.) Since

and

$$
\dot{V}_{1}(k, t)=\dot{x}_{k+1}^{T}(t) P_{1} x_{k+1}(t)+x_{k+1}^{T}(t) P_{1} \dot{x}_{k+1}(t)
$$

$$
\Delta V_{2}(k, t)=y_{k+1}^{T}(t) P_{2} y_{k+1}(t)-y_{k}^{T}(t) P_{2} y_{k}(t)
$$

the associated increment for (16) is

$$
\begin{aligned}
\Delta V(k, t)= & \dot{V}_{1}(k, t)+\Delta V_{2}(k, t) \\
= & \dot{x}_{k+1}^{T}(t) P_{1} x_{k+1}(t)+x_{k+1}^{T}(t) P_{1} \dot{x}_{k+1}(t) \\
& +y_{k+1}^{T}(t) P_{2} y_{k+1}(t)-y_{k}^{T}(t) P_{2} y_{k}(t)
\end{aligned}
$$

which together with (13) and (14) gives

$$
\begin{array}{r}
\Delta V(k, t)=\zeta^{T}(k, t)\left(\left(A_{1}+\Delta A_{1}\right)^{T} P+P\left(A_{1}+\Delta A_{1}\right)\right. \\
\left.+\left(A_{2}+\Delta A_{2}\right) R\left(A_{2}+\Delta A_{2}\right)-R\right) \zeta(k, t)
\end{array}
$$

where

$$
P=\left[\begin{array}{cc}
P_{1} & 0 \\
0 & 0
\end{array}\right], \quad R=\left[\begin{array}{cc}
0 & 0 \\
0 & P_{2}
\end{array}\right]
$$

Hence, stability along the pass holds if $\Delta V(k, t)<0$ for $\zeta(k, t) \neq 0$. Next, the inequality

$$
\Delta V(k, t)+\zeta^{T}(k, t)\left[\begin{array}{cc}
Q_{1} & 0 \\
0 & Q_{2}
\end{array}\right] \zeta(k, t)<0
$$

implies that (9) is stable along the pass. Noting that

$$
\Upsilon=\sum_{k=0}^{\infty} \int_{0}^{\infty}\left(\zeta^{T}(k, t)\left[\begin{array}{cc}
Q_{1} & 0 \\
0 & Q_{2}
\end{array}\right] \zeta(k, t)\right) d t
$$

and, since the process is stable along the pass, we now have that

$$
\begin{aligned}
\Upsilon \leq & -\sum_{k=0}^{\infty} \int_{0}^{\infty}\left(\dot{V}_{1}(k, t)+\Delta V_{2}(k, t)\right) d t \\
= & -\left.\sum_{k=0}^{\infty} x_{k+1}^{T}(t) P_{1} x_{k+1}(t)\right|_{0} ^{\infty} \\
& -\int_{0}^{\infty}\left(\sum_{k=0}^{\infty}\left(y_{k+1}^{T}(t) P_{2} y_{k+1}(t)-y_{k}^{T}(t) P_{2} y_{k}(t)\right)\right) d t \\
= & \sum_{k=0}^{\infty} x_{k+1}^{T}(0) P_{1} x_{k+1}(0) \\
& -\int_{0}^{\infty}\left(y_{\infty}^{T}(t) P_{2} y_{\infty}(t)-y_{0}^{T}(t) P_{2} y_{0}(t)\right) d t \\
= & \sum_{k=0}^{\infty} x_{k+1}^{T}(0) P_{1} x_{k+1}(0)+\int_{0}^{\infty} y_{0}^{T}(t) P_{2} y_{0}(t) d t .
\end{aligned}
$$

Using (18) and (20), a sufficient condition for stability along the pass which ensures that (12) holds is given by

$$
\begin{aligned}
& \left(\left(A_{1}+\Delta A_{1}\right)^{T} P+P\left(A_{1}+\Delta A_{1}\right)\right. \\
& \left.\quad+\left(A_{2}+\Delta A_{2}\right) S\left(A_{2}+\Delta A_{2}\right)-R+Q\right)<0
\end{aligned}
$$

where $Q=\operatorname{diag}\left\{Q_{1}, Q_{2}\right\}, S=\operatorname{diag}\left\{P_{3}, P_{2}\right\}$, and $P_{3}>0$ are any given matrices of the required dimensions. Next, an obvious application of the Schur complement formula yields (23), shown at the bottom of the following page, where

$$
\Lambda=A^{T} P_{1}+\Delta A^{T} P_{1}+P_{1} A+P_{1} \Delta A+Q_{1}
$$

$$
\left[\begin{array}{ccccc}
-P_{2} & P_{2} C & P_{2} D_{0} & P_{2} H_{2} & P_{2} H_{2} \\
C^{T} P_{2} & A^{T} P_{1}+P_{1} A+Q_{1}+\epsilon E_{1}^{T} E_{1} & P_{1} B_{0} & P_{1} H_{1} & P_{1} H_{1} \\
D_{0}^{T} P_{2} & B_{0}^{T} P_{1} & -P_{2}+Q_{2}+\epsilon E_{2}^{T} E_{2} & 0 & 0 \\
H_{2}^{T} P_{2} & H_{1}^{T} P_{1} & 0 & -\epsilon I & 0 \\
H_{2}^{T} P_{2} & H_{1}^{T} P_{1} & 0 & 0 & -\epsilon I
\end{array}\right]<0
$$


On removing the block $-P_{3}$, which is always negative definite, (23) gives the equivalent condition

$$
\begin{aligned}
& {\left[\begin{array}{ccc}
-P_{2} & P_{2} C & P_{2} D_{0} \\
C^{T} P_{2} & A^{T} P_{1}+P_{1} A+Q_{1} & P_{1} B_{0} \\
D_{0}^{T} P_{2} & B_{0}^{T} P_{1} & Q_{2}-P_{2}
\end{array}\right]} \\
& +\left[\begin{array}{ccc}
0 & 0 & 0 \\
0 & E_{1}^{T} & 0 \\
0 & 0 & E_{2}^{T}
\end{array}\right] \\
& \times\left[\begin{array}{ccc}
\mathcal{F}^{T} & 0 & 0 \\
0 & \mathcal{F}^{T} & 0 \\
0 & 0 & \mathcal{F}^{T}
\end{array}\right]\left[\begin{array}{ccc}
0 & 0 & 0 \\
H_{2}^{T} P_{2} & H_{1}^{T} P_{1} & 0 \\
H_{2}^{T} P_{2} & H_{1}^{T} P_{1} & 0
\end{array}\right] \\
& +\left[\begin{array}{ccc}
0 & P_{2} H_{2} & P_{2} H_{2} \\
0 & P_{1} H_{1} & P_{1} H_{1} \\
0 & 0 & 0
\end{array}\right]\left[\begin{array}{ccc}
\mathcal{F} & 0 & 0 \\
0 & \mathcal{F} & 0 \\
0 & 0 & \mathcal{F}
\end{array}\right] \\
& \times\left[\begin{array}{ccc}
0 & 0 & 0 \\
0 & E_{1} & 0 \\
0 & 0 & E_{2}
\end{array}\right]<0
\end{aligned}
$$

and, by an obvious application of the result of Lemma 1, we obtain

$$
\begin{gathered}
{\left[\begin{array}{ccc}
-P_{2} & P_{2} C & P_{2} D_{0} \\
C^{T} P_{2} & \Theta & P_{1} B_{0} \\
D_{0}^{T} P_{2} & B_{0}^{T} P_{1} & Q_{2}-P_{2}+\epsilon E_{2}^{T} E_{2}
\end{array}\right]} \\
+\epsilon^{-1}\left[\begin{array}{ccc}
0 & P_{2} H_{2} & P_{2} H_{2} \\
0 & P_{1} H_{1} & P_{1} H_{1} \\
0 & 0 & 0
\end{array}\right] \\
\quad \times\left[\begin{array}{ccc}
0 & 0 & 0 \\
H_{2}^{T} P_{2} & H_{1}^{T} P_{1} & 0 \\
H_{2}^{T} P_{2} & H_{1}^{T} P_{1} & 0
\end{array}\right]<0
\end{gathered}
$$

where

$$
\Theta=A^{T} P_{1}+P_{1} A+\epsilon E_{1}^{T} E_{1}+Q_{1} .
$$

Finally, an obvious application of the Schur complement formula gives (11) and the proof is complete.

Remark 2: Note that it is possible to minimize the upperbound on the cost function (12) using the following optimization procedure:

$$
\begin{gathered}
\min \left[\sum_{k=0}^{\infty} x_{k+1}^{T}(0) P_{1} x_{k+1}(0)+\int_{0}^{\infty} y_{0}^{T}(t) P_{2} y_{0}(t) d t\right] \\
=\min \left[\sum_{k=0}^{\infty} \operatorname{trace}\left(P_{1} x_{k+1}(0) x_{k+1}^{T}(0)\right)\right. \\
\left.+\operatorname{trace}\left(P_{2} \int_{0}^{\infty} y_{0}(t) y_{0}^{T}(t) d t\right)\right]
\end{gathered}
$$

subject to (11).

\section{Static FeEdBack ConTrol}

The control law (see [2] for a more complete discussion) considered in previous work has the following form over $0 \leq t \leq \alpha$, $k \geq 0$ :

$$
u_{k+1}(t)=\left[\begin{array}{ll}
K_{1} & K_{2}
\end{array}\right]\left[\begin{array}{c}
x_{k+1}(t) \\
y_{k}(t)
\end{array}\right]
$$

where $K_{1}$ and $K_{2}$ are appropriately dimensioned matrices to be designed. In effect, this control law uses feedback of the current state vector (which is assumed to be available for use) and "feedforward" of the previous pass profile vector. Note that in repetitive processes the term "feedforward" is used to describe the case where state or pass profile information from the previous pass (or passes) is used as (part of) the input to a control law applied on the current pass, i.e., to information which is propagated in the pass-to-pass $(k)$ direction.

Applying this control law to (2) yields the closed-loop process state space model

$$
\begin{aligned}
& {\left[\begin{array}{c}
\dot{x}_{k+1}(t) \\
y_{k+1}(t)
\end{array}\right]=\left(\left[\begin{array}{cc}
A+B K_{1} & B_{0}+B K_{2} \\
C+D K_{1} & D_{0}+D K_{2}
\end{array}\right]\right.} \\
& \left.\quad+\left[\begin{array}{cc}
\Delta A+\Delta B K_{1} & \Delta B_{0}+\Delta B K_{2} \\
\Delta C+\Delta D K_{1} & \Delta D_{0}+\Delta D K_{2}
\end{array}\right]\right)\left[\begin{array}{c}
x_{k+1}(t) \\
y_{k}(t)
\end{array}\right]
\end{aligned}
$$

and the associated cost function is

$$
\begin{aligned}
& J=\sum_{k=0}^{\infty} \int_{0}^{\infty}\left(\left[\begin{array}{c}
x_{k+1}(t) \\
y_{k}(t)
\end{array}\right]^{T}\right. \\
& \left.\times\left[\begin{array}{cc}
Q_{1}+K_{1}^{T} \Psi K_{1} & K_{1}^{T} \Psi K_{2} \\
K_{2}^{T} \Psi K_{1} & Q_{2}+K_{2}^{T} \Psi K_{2}
\end{array}\right]\left[\begin{array}{c}
x_{k+1}(t) \\
y_{k}(t)
\end{array}\right]\right) d t .
\end{aligned}
$$

Theorem 3: A differential linear repetitive process described by (2) is robustly stable under the control law (28) if there exist matrices $W_{1}>0, W_{2}>0, N_{1}$, and $N_{2}$ and a scalar $\epsilon>0$ such that the LMI given in (31), shown at the bottom of the following page, holds, where $\Phi=W_{1} A^{T}+A W_{1}+N_{1}^{T} B^{T}+B N_{1}+$ $2 \epsilon H_{1} H_{1}^{T}$ and $\Psi>0, Q_{1}>0$, and $Q_{2}>0$ are the given matrices for the cost function (7). Also, if this condition holds, then stabilizing control law matrices $K_{1}$ and $K_{2}$ are given by

$$
K_{1}=N_{1} W_{1}^{-1}, \quad K_{2}=N_{2} W_{2}^{-1}
$$

and the cost function (30) of the closed-loop process (29) satisfies the following upperbound:

$$
J \leq \sum_{k=0}^{\infty} x_{k+1}^{T}(0) W_{1}^{-1} x_{k+1}(0)+\int_{0}^{\infty} y_{0}^{T}(t) W_{2}^{-1} y_{0}(t) d t .
$$

Proof: Based on (11), we conclude that the closed-loop process (29) is robustly stabilized by the control law (28) if the

$$
\left[\begin{array}{cccc}
-P_{3} & (\star) & (\star) & (\star) \\
0 & -P_{2} & (\star) & (\star) \\
0 & C^{T} P_{2}+\Delta C^{T} P_{2} & \Lambda & (\star) \\
0 & D_{0}^{T} P_{2}+\Delta D_{0}^{T} P_{2} & B_{0}^{T} P_{1}+\Delta B_{0}^{T} P_{1} & Q_{2}-P_{2}
\end{array}\right]<0
$$


matrix inequality given in (34), shown at the bottom of the page, is satisfied, where

$$
\begin{aligned}
& \Psi_{1}=A^{T} P_{1}+P_{1} A+K_{1}^{T} B^{T} P_{1}+P_{1} B K_{1}+Q_{1}+K_{1}^{T} \Psi K_{1} \\
& \Psi_{2}=-P_{2}+Q_{2}+K_{2}^{T} \Psi K_{2} \\
& \Psi_{3}=\Delta A^{T} P_{1}+P_{1} \Delta A+K_{1}^{T} \Delta B^{T} P_{1}+P_{1} \Delta B K_{1} .
\end{aligned}
$$

Now set $W_{1}=P_{1}^{-1}, W_{2}=P_{2}^{-1}, U_{1}=W_{1} Q_{1} W_{1}$, and $U_{2}=$ $W_{2} Q_{2} W_{2}$ and then premultiply and postmultiply both sides of this last inequality by $\operatorname{diag}\left\{W_{2}, W_{1}, W_{2}\right\}$ to obtain (35), shown at the bottom of the page, where

$$
\begin{aligned}
\Psi_{4}= & W_{1} A^{T}+A W_{1}+W_{1} K_{1}^{T} B^{T} \\
& +B K_{1} W_{1}+U_{1}+W_{1} K_{1}^{T} \Psi K_{1} W_{1} \\
\Psi_{5}= & -W_{2}+U_{2}+W_{2} K_{2}^{T} \Psi K_{2} W_{2} \\
\Psi_{6}= & \Delta W_{1} A^{T}+\Delta A W_{1} \\
& +W_{1} K_{1}^{T} \Delta B^{T}+\Delta B K_{1} W_{1} .
\end{aligned}
$$

This last inequality can be rewritten as (36), shown at the bottom of the page, where

$$
\begin{aligned}
\Psi_{7}= & W_{1} A^{T}+A W_{1}+W_{1} K_{1}^{T} B^{T}+B K_{1} W_{1} \\
& +U_{1}+W_{1} K_{1}^{T} \Psi K_{1} W_{1} \\
\Psi_{8}= & -W_{2}+U_{2}+W_{2} K_{2}^{T} \Psi K_{2} W_{2}
\end{aligned}
$$

and, by an obvious application of the result of Lemma 1, we now obtain

$$
\begin{aligned}
& \begin{array}{c}
-W_{2} \\
W_{1} C^{T}+N_{1}^{T} D^{T} \\
W_{2} D_{0}^{T}+N_{2}^{T} D^{T} \\
W_{2} B_{0}^{T}+N_{2}^{T} B^{T}+N^{T} \\
+\epsilon\left[\begin{array}{ccc}
0 & H_{2} & H_{2} \\
0 & H_{1} & H_{1} \\
0 & 0 & 0
\end{array}\right]\left[\begin{array}{ccc}
0 & 0 & 0 \\
H_{2}^{T} & H_{1}^{T} & 0 \\
H_{2}^{T} & H_{1}^{T} & 0
\end{array}\right]
\end{array} \\
& \begin{array}{l}
+\epsilon\left[\begin{array}{ccc}
0 & H_{2} & H_{2} \\
0 & H_{1} & H_{1} \\
0 & 0 & 0
\end{array}\right]\left[\begin{array}{ccc}
0 & 0 & 0 \\
H_{2}^{T} & H_{1}^{T} & 0 \\
H_{2}^{T} & H_{1}^{T} & 0
\end{array}\right] \\
+\epsilon^{-1}\left[\begin{array}{ccc}
0 & 0 & 0 \\
0 & N_{1}^{T} E_{3}^{T}+W_{2} E_{1}^{T} & 0 \\
0 & 0 & N_{2}^{T} E_{3}^{T}+W_{2} E_{2}^{T}
\end{array}\right]
\end{array} \\
& \times\left[\begin{array}{ccc}
0 & 0 & 0 \\
0 & E_{1} W_{1}+E_{3} N_{1} & 0 \\
0 & 0 & E_{2} W_{2}+E_{3} N_{2}
\end{array}\right]<0
\end{aligned}
$$

$$
\left[\begin{array}{cccccccc}
-W_{2}+2 \epsilon H_{2} H_{2}^{T} & (\star) & (\star) & (\star) & (\star) & (\star) & (\star) & (\star) \\
W_{1} C^{T}+N_{1}^{T} D^{T}+2 \epsilon H_{1} H_{2}^{T} & \Phi & (\star) & (\star) & (\star) & (\star) & (\star) & (\star) \\
W_{2} D_{0}^{T}+N_{2}^{T} D^{T} & W_{2} B_{0}^{T}+N_{2}^{T} B^{T} & -W_{2} & (\star) & (\star) & (\star) & (\star) & (\star) \\
0 & E_{1} W_{1}+E_{3} N_{1} & 0 & -\epsilon I & (\star) & (\star) & (\star) & (\star) \\
0 & 0 & E_{2} W_{2}+E_{3} N_{2} & 0 & -\epsilon I & (\star) & (\star) & (\star) \\
0 & N_{1} & N_{2} & 0 & 0 & -\Psi^{-1} & (\star) & (\star) \\
0 & W_{1} & 0 & 0 & 0 & 0 & -Q_{1}^{-1} & (\star) \\
0 & 0 & W_{2} & 0 & 0 & 0 & 0 & -Q_{2}^{-1}
\end{array}\right]
$$

$$
\left[\begin{array}{ccc}
-P_{2} & (\star) & (\star) \\
C^{T} P_{2}+K_{1}^{T} D^{T} P_{2} & \Psi_{1} & (\star) \\
D_{0}^{T} P_{2}+K_{2}^{T} D^{T} P_{2} & B_{0}^{T} P_{1}+K_{2}^{T} B^{T} P_{1}+K_{2}^{T} \Psi K_{1} & \Psi_{2}
\end{array}\right]+\left[\begin{array}{ccc}
0 & (\star) & (\star) \\
\Delta C^{T} P_{2}+K_{1}^{T} \Delta D^{T} P_{2} & \Psi_{3} \\
\Delta D_{0}^{T} P_{2}+K_{2}^{T} \Delta D^{T} P_{2} & \Delta B_{0}^{T} P_{1}+K_{2}^{T} \Delta B^{T} P_{1} & (\star) \\
0
\end{array}\right]<0
$$

$$
\begin{aligned}
& {\left[\begin{array}{ccc}
-W_{2} & (\star) & (\star) \\
W_{1} C^{T}+W_{1} K_{1}^{T} D^{T} & \Psi_{4} & (\star) \\
W_{2} D_{0}^{T}+W_{2} K_{2}^{T} D^{T} & W_{2} B_{0}^{T}+W_{2} K_{2}^{T} B^{T}+W_{2} K_{2}^{T} \Psi K_{1} W_{1} & \Psi_{5}
\end{array}\right]} \\
& +\left[\begin{array}{ccc}
0 & (\star) & (\star) \\
W_{1} \Delta C^{T}+W_{1} K_{1}^{T} \Delta D^{T} & \Psi_{6} & (\star) \\
W_{2} \Delta D_{0}^{T}+W_{2} K_{2}^{T} \Delta D^{T} & W_{2} \Delta B_{0}^{T}+W_{2} K_{2}^{T} \Delta B^{T} & 0
\end{array}\right]<0
\end{aligned}
$$

$$
\begin{aligned}
& {\left[\begin{array}{ccc}
-W_{2} & (\star) & (\star) \\
W_{1} C^{T}+W_{1} K_{1}^{T} D^{T} & \Psi_{7} & (\star) \\
W_{2} D_{0}^{T}+W_{2} K_{2}^{T} D^{T} & W_{2} B_{0}^{T}+W_{2} K_{2}^{T} B^{T}+W_{2} K_{2}^{T} \Psi K_{1} W_{1} & \Psi_{8}
\end{array}\right]} \\
& +\left[\begin{array}{ccc}
0 & H_{2} & H_{2} \\
0 & H_{1} & H_{1} \\
0 & 0 & 0
\end{array}\right]\left[\begin{array}{ccc}
\mathcal{F} & 0 & 0 \\
0 & \mathcal{F} & 0 \\
0 & 0 & \mathcal{F}
\end{array}\right]\left[\begin{array}{ccc}
0 & 0 & 0 \\
0 & E_{1} W_{1}+E_{3} K_{1} W_{1} & 0 \\
0 & 0 & E_{2} W_{2}+E_{3} K_{2} W_{2}
\end{array}\right]
\end{aligned}
$$

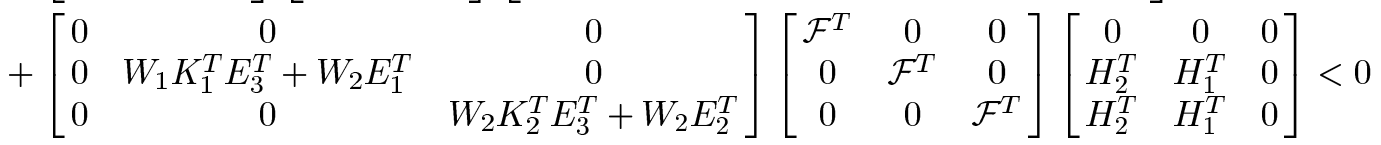


where

$$
\begin{aligned}
\Psi_{9} & =W_{1} A^{T}+A W_{1}+N_{1}^{T} B^{T}+B N_{1}+U_{1}+N_{1}^{T} \Psi N_{1} \\
\Psi_{10} & =-W_{2}+U_{2}+N_{2}^{T} \Psi N_{2}
\end{aligned}
$$

and $N_{1}=K_{1} W_{1}$ and $N_{2}=K_{2} W_{2}$. Finally, making an obvious application of the Schur complement formula gives (31), and the proof is complete.

Remark 3: Note that it is possible to minimize the upperbound on the cost function (12) using the following optimization procedure:

$$
\begin{gathered}
\min \left[\sum_{k=0}^{\infty} x_{k+1}^{T}(0) W_{1}^{-1} x_{k+1}(0)+\int_{0}^{\infty} y_{0}^{T}(t) W_{2}^{-1} y_{0}(t) d t\right] \\
=\min \left[\sum_{k=0}^{\infty} \operatorname{trace}\left(W_{1}^{-1} x_{k+1}(0) x_{k+1}^{T}(0)\right)\right. \\
\left.\quad+\operatorname{trace}\left(W_{2}^{-1} \int_{0}^{\infty} y_{0}(t) y_{0}^{T}(t) d t\right)\right]
\end{gathered}
$$

subject to (31). This convex optimization algorithm cannot be applied in this case of Theorem 3 because of the nonlinear terms $W_{1}^{-1}$ and $W_{2}^{-1}$. However, a suboptimal controller can be achieved by the following algorithm (see also Remark 1).

First, assume there exists a scalar $\sigma>0$ and matrices $\Sigma$ and $\Omega$ which satisfy

$$
\begin{aligned}
\sigma & >\sum_{k=0}^{p} x_{k+1}^{T}(0) W^{-1} x_{k+1}(0), \\
\Sigma \Sigma^{T} & =\int_{0}^{\alpha} y_{0}(t) y_{0}^{T}(t) d t \\
\Sigma^{T} W_{2}^{-1} \Sigma & <\Omega
\end{aligned}
$$

and hence we can write

$$
\begin{aligned}
\int_{0}^{\alpha} y_{0}^{T}(t) W_{2}^{-1} y_{0}(t) d t & =\operatorname{trace}\left(\Sigma \Sigma^{T} W_{2}^{-1}\right) \\
& =\operatorname{trace}\left(\Sigma^{T} W_{2}^{-1} \Sigma\right) \\
& <\operatorname{trace}(\Omega)
\end{aligned}
$$

Next, an obvious application of the Schur complement formula gives

$$
\left[\begin{array}{cc}
-\beta & x_{k+1}^{T}(0) \\
x_{k+1}(0) & -W_{1}
\end{array}\right]>0 \text { and }\left[\begin{array}{cc}
-\Omega & \Sigma^{T} \\
\Sigma & -W_{2}
\end{array}\right]>0
$$

respectively. Finally, the following minimization problem can be formulated:

$$
\min (\beta+\operatorname{trace}(\Omega))
$$

subject to: (31) and (41), and the solution (32) now guarantees that the cost function is minimized over the finite pass length in the case when only a finite number of trials is actually completed.

\section{CONCluSION}

In this paper, the guaranteed cost control problem for differential repetitive processes in the presence of norm-bounded uncertainty has been solved. Space considerations preclude the inclusion of numerical examples, but it can be stated that an extensive range of these have been computed with a very high degree of numerical reliability evident. Of course, the results given here are based on a sufficient, but not necessary, stability condition and hence there could well be a considerable degree of conservativeness present in at least some cases. At present, however, we argue that this setting is the only one which allows controller design, and, if alternatives are developed, then these results will serve as a benchmark for comparative purposes. Finally, it should be possible to extend the analysis here to other performance control problems where performance is measured by an appropriately defined cost function or index.

\section{REFERENCES}

[1] N. Amann, D. H. Owens, and E. Rogers, "Predictive optimal iterative learnig control," Int. J. Control, vol. 69, pp. 203-226, 1998.

[2] K. G. Kowski, W. Paszke, E. Rogers, S. Xu, J. Lam, and D. Owens, "Stability and control of differential linear repetitive processes using an lmi seting," IEEE Trans. Circ. Syst. II, vol. 50, pp. 662-666, Sept. 2003.

[3] X. Guan, C. Long, and G. Duan, "Robust optimal guaranteed cost control for 2D discrete systems," IEE Proc.-Control Theory Appl., vol. 148, no. 5, pp. 355-361, 2001.

[4] P. P. Khargonekar, I. R. Petersen, and K. Zhou, "Robust stabilization of uncertain linear systems: Quadratic stabilizability and $H_{\infty}$ control theory," IEEE Trans. Automat. Contr., vol. 35, pp. 356-361, Mar. 1990.

[5] Y. Nesterov and A. Nemirovskii, Interior-Point Polynomial Algorithms in Convex Programing. Philadelphia, PA: SIAM, 1994, vol. 13, SIAM Studies in Applied Mathematics.

[6] P. D. Roberts, "Numerical investigations of a stability theorem arising from 2-dimensional analysis of an iterative optimal control algorithm," Multidimensional Syst. Signal Process., vol. 11, no. 1/2, pp. 109-124, 2000.

[7] E. Rogers and D. H. Owens, Stability Analysis for Linear Repetitive Processes. Berlin, Germany: Springer-Verlag, 1992, vol. 175, Lecture Notes in Control and Information Sciences. 